\title{
1 Electron extraction mechanisms of a micro-ECR neutralizer
}

3 Yoshinori Takao ${ }^{1 *}$, Kenta Hiramoto $^{2}$, Yuichi Nakagawa ${ }^{3}$, Yusuke Kasagi ${ }^{3}$, Hiroyuki Koizumi ${ }^{4}$,

4 and Kimiya Komurasaki ${ }^{3}$

$5 \quad{ }^{1}$ Division of Systems Research, Yokohama National University, Yokohama 240-8501, Japan

$6{ }^{2}$ Department of Systems Integration, Yokohama National University, Yokohama 240-8501,

7 Japan

$8{ }^{3}$ Department of Aeronautics and Astronautics, The University of Tokyo, Bunkyo, Tokyo 1139 8656, Japan

$10{ }^{4}$ Department of Advanced Energy, The University of Tokyo, Kashiwa, Chiba 277-8561, Japan

11 E-mail: takao@ynu.ac.jp

13 Abstract

14 Three-dimensional particle simulations have been conducted to analyze the mechanisms of 15 electron extraction through the orifices of a $4.2 \mathrm{GHz}$ microwave discharge microneutralizer, 16 using a xenon electron cyclotron resonance plasma. The dimensions of the neutralizer are $1720 \times 20 \times 4 \mathrm{~mm}^{3}$, and a ring-shaped microwave antenna and permanent magnets are employed for 18 its discharges. The numerical model is composed of a particle-in-cell simulation with a Monte

19 Carlo collision algorithm for charged particle motions, a finite-difference time-domain method 20 for microwaves, and a finite element analysis for magnetostatic fields. The simulation results 
21 have shown that the electrostatic field inside the plasma source has a dominant effect on 22 electron extraction. The extracted electrons move along the magnetic field line to the orifice 23 entrances and the $\boldsymbol{E} \times \boldsymbol{B}$ drift at the orifice edge induces electron extraction. 


\section{Introduction}

Electron sources are widely used in various fields from basic sciences to industrial applications, such as $\gamma$-ray sources, ${ }^{1)}$ high-current electron beams for microwave tubes, ${ }^{2)}$ inductively coupled plasma mass spectrometry, ${ }^{3)}$ etching tools, ${ }^{4)}$ welding flow lines, ${ }^{5)}$ and neutralization of ion beams for ion and Hall thrusters. ${ }^{6)}$ Neutralization is required for not only ion and Hall thrusters but also a low-energy ion implantation along with shrinkage of transistors to cancel out the space charge effect for shallow depths of implants. ${ }^{7)}$ Most electron sources are generated by plasma discharges, and a micro-electron source, a neutralizer which we report here, also employs a plasma discharge sustained by electron cyclotron resonance (ECR).

The micro-ECR neutralizer presented here was developed by the University of Tokyo, and it is one of the components of a miniature ion propulsion system (MIPS) for a 50-kg-class microspacecraft, HODOYOSHI-4. ${ }^{8)}$ The microspacecraft was launched on June 19, 2014 and the MIPS was operated successfully in space on October 28, 2014 for the first time in the world. The neutralizer of the MIPS is based on a low-power microwave neutralizer for the 150-mAclass ion beam exhausted from an ion thruster, ${ }^{9-11)}$ and employs the same frequency of $4.2 \mathrm{GHz}$ and ring-shaped permanent magnets, but a different type of microwave antenna is used because of the small size of the MIPS neutralizer. ${ }^{12)}$

Although the MIPS has already been operated in space, the mechanism of electron extraction from its neutralizer is still unclear and needs to be elucidated for a better performance.

Owing to its small size, the neutralizer operates using an identical discharge chamber, the same 
microwave power, and a half gas flow rate compared with the ion source, which indicates that the MIPS neutralizer consumes resources (space, power, and propellant) more significantly than conventional ion propulsion systems. One of the reasons for its poor performance is considered to be the magnetic confinement of electrons inside the plasma source. Magnetic confinement is necessary for good plasma production to reduce the loss of plasma toward the chamber wall. The confinement, however, leads to a negative effect on electron extraction. Hence, it is important to investigate the process through which electrons are extracted from the plasma source with applied magnetic fields.

In order to elucidate the extraction mechanism for a better performance of the neutralizer, numerical simulations could be a powerful tool to compensate for the lack of information obtained from experiments. Hence, we have developed a three-dimensional numerical model, which consists of a particle-in-cell simulation with a Monte Carlo collision algorithm (PIC-MCC) for the kinetics of charged particles, ${ }^{13)}$ a finite-difference time-domain (FDTD) algorithm for the electromagnetic fields of microwaves, ${ }^{14)}$ and a finite element analysis for the magnetostatic fields of permanent magnets. The numerical analysis was performed for the plasma source, ${ }^{15)}$ and now is being used for the study on electron extraction. Numerical results were compared with experimental data, in which the current density distribution on the discharge chamber wall was obtained, and showed a reasonable agreement. ${ }^{16,17)}$

In the present work, to investigate the mechanisms of electron extraction from the micro-ECR neutralizer of the MIPS, we have conducted three-dimensional PIC-MCC 
simulations and focused mainly on the electron trajectories extracted from the plasma source to the outside (vacuum) through orifices. This paper is an extended version of our conference proceedings paper. ${ }^{18)}$ In the next section, we briefly describe our numerical model, ${ }^{15)}$ which was extended to three dimensions from two axisymmetric dimensions for a micro-RF ion thruster, ${ }^{19)}$ and a miniature inductively coupled plasma source. ${ }^{20,21)}$ In Sect. 3, we present the results of macroscopic parameters and electron trajectories, which show that the self-generated electrostatic field inside the plasma source is the most important factor and the $\boldsymbol{E} \times \boldsymbol{B}$ drift velocity at the orifice edges is one of the possible mechanisms to extract electrons, which is newly added in the present paper. Finally, conclusions are drawn of this paper in Sect. 4.

\section{Numerical model}

The three-dimensional PIC-MCC model described in our previous paper was employed under the following assumptions. ${ }^{15)}$ (i) Only singly ionized xenon and electrons are treated as particles. The reactions taken into account are elastic scattering, excitation, and ionization for electrons, ${ }^{22-}$ ${ }^{24)}$ and elastic scattering and charge exchange for ions, ${ }^{25)}$ where the null-collision method is employed to reduce the calculation time. ${ }^{26)}$ The motion of excited-state atoms and Coulomb collisions are not taken into account. Neutral particles are assumed to be spatially uniform throughout the simulation and have a Maxwellian velocity distribution at a gas temperature of $300 \mathrm{~K}$. (ii) The magnetic fields of microwaves are neglected compared with the magnetostatic fields of the permanent magnets. (iii) The effect of plasma current is not taken into account 
owing to the low power of microwaves in this study. ${ }^{27)}$

The numerical simulations were carried out as shown in Fig. 1. First, we set the initial conditions and then solve Maxwell's equations by FDTD for the electromagnetic fields of microwaves with a time increment $\Delta t_{\mathrm{EM}}=2.98 \times 10^{-13} \mathrm{~s}(1 / 800$ of a microwave cycle for 4.2 $\mathrm{GHz}$ ) until we obtain a steady-state solution without plasma. We use the amplitude of the electric fields of microwaves, $\boldsymbol{E}_{0}$, in the steady state for the PIC-MCC calculations as described below. Second, electrostatic PIC-MCC calculations are conducted with a time step $\Delta t_{\mathrm{e}}=$ $5.95 \times 10^{-12}$ s (1/40 of a microwave cycle) using the electrostatic field $\boldsymbol{E}_{\mathrm{ES}}$ of the plasma, the time-varying electric field of microwaves $\boldsymbol{E}_{\mathrm{EM}}=\boldsymbol{E}_{0} \cos (\omega t)$, and the magnetostatic fields $\boldsymbol{B}_{\mathrm{st}}$ generated by the permanent magnets, where $\omega$ is the angular frequency of microwaves and $\boldsymbol{B}_{\mathrm{st}}$ is determined using ANSYS Emag ${ }^{\mathrm{TM}}$ software. Here, in order to speed up the simulation, the motion of ions is updated with a time step $\Delta t_{\mathrm{i}}=2.38 \times 10^{-10} \mathrm{~s}$ (one microwave cycle) by neglecting $\boldsymbol{E}_{\mathrm{EM}}$ and using the time-averaged $\boldsymbol{E}_{\mathrm{ES}}$ over one microwave cycle because the frequency of $4.2 \mathrm{GHz}$ is much higher than the ion plasma frequency. In the simulation, the power absorbed in the plasma $P_{\text {abs }}$ is used as an input parameter, where $P_{\text {abs }}$ is obtained by calculating the change in the kinetic energy of electrons and ions before and after the calculation of the equation of motion. ${ }^{28)}$ Last, we rescale the amplitude $\boldsymbol{E}_{0}$ to yield the specified power absorbed in the plasma $P_{0}$ and iterate the above procedure until the steady-state solution is obtained.

Figure 2 shows the computational domain and dimensions for electron extraction in 
105 the present study. The Cartesian coordinate system is employed and its origin is set at the center 106 of the ring-shaped antenna on the interface between the metal wall and the plasma in the $z$

107 direction. The lengths in the $x, y$, and $z$ directions are 20, 20, and $10 \mathrm{~mm}$, respectively, including 108 the region for the investigation of electron extraction. The structure of the antenna and ring109 shaped magnets are the same as those employed in our previous paper, ${ }^{15)}$ in which the 110 dimensions are indicated in detail. We place a 0.6 - $\mathrm{mm}$-thick orifice plate at $z=4.0 \mathrm{~mm}$, where 111 four orifices with a diameter of $2.2 \mathrm{~mm}$ are formed and their centers are located on the circle 112 with a $5.0 \mathrm{~mm}$ radius of the $x-y$ origin at an angular degree of 45 with respect to the $x / y$-axis.

113 The grid spacing is set at $0.2 \mathrm{~mm}$ at regular intervals. For the plasma parameter range calculated

114 in the present study, the grid spacing ( $\Delta x, \Delta y$, and $\Delta z)$ is small enough to satisfy the following 115 condition: the Debye length $\lambda_{\mathrm{D}}$ is roughly larger than $\Delta x / 3, \Delta y / 3$, and $\Delta z / 3 .^{29)}$ As boundary 116 conditions, the potential on the entire metal area is set at zero and we set $20 \mathrm{~V}$ at $\mathrm{z}=10 \mathrm{~mm}$ for 117 electron extraction. All electrons and ions disappear at the walls, antenna, and other boundaries, 118 where no reflection and charge accumulation are assumed.

120 3. Results and discussion

121 A xenon plasma discharge was calculated for the microwave discharge neutralizer under the

122 base case condition; namely, the xenon gas pressure is $1 \mathrm{mTorr}$, the microwave frequency is

$1234.2 \mathrm{GHz}$, and the absorbed power is $0.3 \mathrm{~W}$. The initial densities of both electrons and ions are 124 set at $1.0 \times 10^{16} \mathrm{~m}^{-3}$ and are distributed uniformly in the simulation area. The initial electron and 
ion temperatures are 2.0 and $0.05 \mathrm{eV}$, respectively.

\subsection{Macroscopic parameters}

128 The macroscopic parameters, such as electron density and electron temperature, were

129 determined by averaging over 50000 microwave cycles (11.9 $\mu$ s) after the steady state was

130 reached. The peak plasma density is located in the ECR layer on the right side of the antenna,

131 where the maximum electron density is $1.6 \times 10^{17} \mathrm{~m}^{-3}$, and their distributions spread along the

132 magnetic field lines, producing the ring-shaped profile of plasma density. Such a distribution

133 was also confirmed in the experiment. This result indicates that the plasma is well confined

134 because of the mirror magnetic fields. The distributions of electron temperature and plasma

135 potential are almost the same as the distribution of plasma density, where the peak electron

136 temperature and potential obtained are $16 \mathrm{eV}$ and $22 \mathrm{~V}$, respectively. The details of the other

137 results are described in our previous paper. ${ }^{15)}$

To investigate the mechanism of electron extraction through the orifices of the

139 neutralizer, we have obtained the time-averaged electron current streamlines, as shown in Fig.

1403 , where the distributions of electron current density are also plotted as cross-sectional views.

141 The figure clearly indicates the grad- $B$ and curvature drift of electrons due to the magnetostatic

142 field of the permanent magnets. Some electrons seem to flow back into the plasma source from

143 the outside. Although the circulation of the electrons can be explained by the drift motion, the

144 mechanism of electron motion in the $z$ direction cannot be fully understood unless only the 
time-averaged electron motion is focused on.

\subsection{Force exerted on a single electron}

148 As described in Sect. 2, the motion of electrons are attributed to the sum of the electrostatic

149 electric field $\boldsymbol{E}_{\mathrm{ES}}$, the microwave electromagnetic field $\boldsymbol{E}_{\mathrm{EM}}$, and the magnetostatic field of the

150 permanent magnets $\boldsymbol{B}_{\text {st. }}$ Electron-neutral collisions also affect the electron motion. However,

151 their effect would be relatively small because of few collisions at a low pressure of $1 \mathrm{mTorr}$ in

152 this work. To determine which effect plays a dominant role in extracting electrons from the

153 confined plasma source through the orifice, we have conducted several simulations with

154 deletion of a portion of electromagnetic fields artificially after the steady-state solution is

155 obtained and tracked the motion of electrons. The results are summarized as follows. The

156 magnetostatic field $\boldsymbol{B}_{\text {st }}$ causes only the mirror confinement and grad- $B$ and curvature drift, so

157 that the electron trajectory only shows a circulation, similar to the electron current streamlines,

158 as shown in Fig. 3. Although the addition of $\boldsymbol{E}_{\mathrm{EM}}$ resulted in only a slight change, the addition

159 of $\boldsymbol{E}_{\mathrm{ES}}$ clearly has a strong effect on the electron trajectory inside the plasma source and

160 electrons are extracted from the orifice. Electrons are not extracted unless the effect of $\boldsymbol{E}_{\mathrm{ES}}$ is

161 taken into account in the calculations. ${ }^{16,17)}$

Electrons have to overcome the potential barrier as well as escape from the magnetic

163 confinement in order to be extracted through the orifice. Since the force due to $\boldsymbol{E}_{\mathrm{ES}}$ exerts on

164 electrons in the opposite direction of extraction, the distribution of $\boldsymbol{E}_{\mathrm{ES}}$ does not show a clear 
165

166

167

168

169

170

171

172

173

174

175

176

177

178

179

180

181

182

183

184

reason for extraction from the bulk plasma into the vacuum through the orifice, although $\boldsymbol{E}_{\mathrm{ES}}$ is required for electron extraction. An electron moves along the magnetic field line and travels back and forth as a result of its confinement due to the mirror magnetic fields, as shown in Fig. 2(d). The electron trajectory can move from one of the magnetic field lines to another line when the electron is reflected by the potential barrier in the sheath. If the trajectory was going outward gradually, the electron could be extracted through one of the orifices. ${ }^{16,17)}$

\subsection{Cross-field motion}

Since cross-field motion of grad- $B$ and curvature drift only results in the circulation of electrons along the ring-shaped antenna, as shown in Fig 3, one of possible mechanisms of cross-field motion for electron extraction is the $\boldsymbol{E} \times \boldsymbol{B}$ drift motion. Figure 4 shows the two-dimensional distribution of the time-averaged $\boldsymbol{E} \times \boldsymbol{B}$ drift velocity in the $z$ direction at the orifice plate $(z=$ 4.0 mm), which is obtained from the electric and magnetic fields at the grid points. As clearly seen in the figure, the positive drift velocity is obtained on the clockwise side of the orifice while the negative drift velocity is seen on the counterclockwise side. Since two ring-shaped permanent magnets are employed, the axisymmetric magnetic field is generated with respect to the $x-y$ origin. The direction of the time-averaged electric field inside the orifice is from the center to the edge of the orifice. These magnetic and electric fields produce the drift velocity component at the edge of the orifice.

To investigate the $\boldsymbol{E} \times \boldsymbol{B}$ drift effect, 1000 electrons are sampled at random and then 
185

their trajectories are traced for a time span of 2000 microwave cycles (476 ns). Figure 5 shows a scatter diagram of electrons at the orifice plate $(z=4.0 \mathrm{~mm})$, in which the positions of the electrons having a drift velocity in the outgoing direction from the plasma source to the vacuum are plotted in red while those of electrons having a drift velocity in the incoming direction from the vacuum to the plasma source are plotted in blue. It seems likely that the tendency of electron distribution inside each orifice is quite similar to the time-averaged $\boldsymbol{E} \times \boldsymbol{B}$ drift velocity in the $z$ direction, as shown in Fig. 4. A typical trajectory of a single electron extracted from one of the orifices together with vectors of $\boldsymbol{E} \times \boldsymbol{B}$ drift velocity at some points is shown in Fig. 6, in which the plane is set in such a way that it always passes through both the positions of the electron and the z-axis during the electron tracking. Although the electron does not directly follow the direction of $\boldsymbol{E} \times \boldsymbol{B}$ drift velocity, at least its projected direction is consistent with the electron motion.

Figure 7(a) shows the number of outgoing and incoming electrons passing through different regions inside the orifices; the regions are defined in Fig. 7(b). Each region is divided by lines passing through the $x-y$ origin at regular intervals of five angular degrees. While the number of incoming electrons are larger than that of outgoing electrons at 50 angular degrees or larger (on the counterclockwise side of the orifice), the number of outgoing electrons are larger than that of incoming electrons at less than 50 angular degrees (on the clockwise side). This tendency is consistent with the distribution of the time-averaged $\boldsymbol{E} \times \boldsymbol{B}$ drift velocity in the $z$ direction, as shown in Fig. 4. The plasma density inside the plasma source is much higher 
205 than that in the vacuum area; thus, the number of outgoing electrons through the orifice region 206 below 40 angular degrees are larger than that of incoming electrons through the orifice region 207 over 50 angular degrees, although both regions have the same area and the same magnitude of 208 force is exerted. However, there are almost no force that induces the $\boldsymbol{E} \times \boldsymbol{B}$ drift in the central 209 area of the orifice, where the largest number of electrons is extracted from the plasma source to 210 the vacuum. This extraction mechanism cannot be explained by the $\boldsymbol{E} \times \boldsymbol{B}$ drift motion only and 211 is still left for future work.

\section{Conclusions}

214 We have conducted three-dimensional PIC-MCC simulations to investigate the mechanisms of 215 electron extraction for a micro-ECR neutralizer. The simulations have shown that electrons are 216 not extracted unless the effect of $\boldsymbol{E}_{\mathrm{ES}}$ is taken into account; thus, the electron extraction is 217 mainly attributed to the self-generated electrostatic field inside the plasma source, even though $218 \boldsymbol{E}_{\mathrm{ES}}$ gives the opposite force of electron extraction from the bulk plasma toward the orifice plate.

219 If the electrons are trapped in the magnetic field passing close to the orifice, such electrons can 220 move to just before the orifice because of magnetic confinement. Although the extraction 221 mechanism at the central area of the orifice is still not clear, the $\boldsymbol{E} \times \boldsymbol{B}$ drift provides the 222 mechanism by which electrons are extracted from the plasma source to the outside at the orifice 223 edge. 


\section{Acknowledgments}

226 This work was financially supported in part by a Grant-in-Aid for Scientific Research (B)

227 (Grant No. 25289304) from the Japan Society for the Promotion of Science. Part of the 228 computer simulations was performed on the KDK computer system at the Research Institute 229 for Sustainable Humanosphere, Kyoto University.

230

231

232 


\section{References}

234 1) Y. Glinec, J. Faure, L. L. Dain, S. Darbon, T. Hosokai, J. J. Santos, E. Lefebvre, J. P. Rousseau, F. Burgy, B. 235 Mercier, and V. Malka, Phys. Rev. Lett. 94, 025003 (2005).

236 2) A. Krokhmal, J. Z. Gleizer, Y. E. Krasik, J. Felsteiner, and V. I. Gushenets, J. Appl. Phys. 94, 44 (2003).

237 3) N. Praphairaksit and R. S. Houk, Anal. Chem. 72, 4435 (2000).

238 4) P. L. G. Ventzek, M. Grapperhaus, and M. J. Kushner, J. Vac. Sci. Technol. B 12, 3118 (1994).

239 5) I. Osipov and N. Rempe, Rev. Sci. Instrum. 71, 1638 (2000).

240 6) D. M. Goebel and I. Katz, Fundamentals of Electric Propulsion: Ion and Hall Thrusters (Wiley, Hoboken, $241 \quad$ NJ, 2008).

242 7) M. Sugitani, Rev. Sci. Instrum. 85, 02C315 (2014).

243 8) H. Koizumi, K. Komurasaki, and Y. Arakawa, 48th AIAA/ASME/SAE/ASEE Joint Propulsion Conf. Exhibit, 244 2012, AIAA-2012-3949.

245 9) I. Funaki and H. Kuninaka, Jpn. J. Appl. Phys. 40, 2495 (2001).

246 10) H. Kuninaka, K. Nishiyama, I. Funaki, T. Yamada, Y. Shimizu, and J. Kawaguchi, J. Propul. Power 23, 544 247 (2007).

248 11) H. Kuninaka and S. Satori, J. Propul. Power 14, 1022 (1998).

249 12) H. Koizumi and H. Kuninaka, J. Propul. Power 26, 601 (2010).

250 13) C. K. Birdsall, IEEE Trans. Plasma Sci. 19, 65 (1991).

251 14) K. Yee, IEEE Trans. Antennas Propag. 14, 302 (1966).

252 15) Y. Takao, H. Koizumi, K. Komurasaki, K. Eriguchi, and K. Ono, Plasma Sources Sci. Technol. 23, 064004 $253 \quad$ (2014)

254 16) Y. Takao, H. Koizumi, Y. Kasagi, and K. Komurasaki, to be published in Trans. JSASS Aerospace Tech. Japan.

255 17) Y. Takao, H. Koizumi, Y. Kasagi, and K. Komurasaki, Joint Conf. 30th Int. Symp. Space Technology and 256 Science / 34th Int. Electric Propulsion Conf. / 6th Nano-satellite Symp., 2015, IEPC-2015-159/ISTS-2015-b159.

18) Y. Takao, K. Hiramoto, Y. Nakagawa, Y. Kasagi, H. Koizumi, and K. Komurasaki, 9th Int. Conf. Reactive Plasmas / 68th Gaseous Electronics Conf. / 33rd Symp. Plasma Processing, 2015, GT1.00173. 
260 19) Y. Takao, M. Sakamoto, K. Eriguchi, and K. Ono, Trans. JSASS Aerospace Tech. Japan 12, Pb_13 (2014).

261 20) Y. Takao, K. Eriguchi, and K. Ono, J. Appl. Phys. 112, 093306 (2012).

262 21) Y. Takao, N. Kusaba, K. Eriguchi, and K. Ono, J. Appl. Phys. 108, 093309 (2010).

263 22) M. Hayashi, J. Phys. D 16, 581 (1983).

264 23) F. J. d. Heer, R. H. J. Jansen, and W. v. d. Kaay, J. Phys. B 12, 979 (1979).

265 24) D. Rapp and P. Englander-Golden, J. Chem. Phys. 43, 1464 (1965).

266 25) I. D. Boyd, J. Appl. Phys. 95, 4575 (2004).

267 26) V. Vahedi and M. Surendra, Comput. Phys. Commun. 87, 179 (1995).

268 27) Y. Takao, K. Eriguchi, K. Ono, Y. Sugita, H. Koizumi, and K. Komurasaki, 50th AIAA/ASME/SAE/ASEE 269 Joint Propulsion Conf., 2014, AIAA-2014-3829.

270 28) M. Surendra and D. B. Graves, IEEE Trans. Plasma Sci. 19, 144 (1991).

271 29) C. K. Birdsall and A. B. Langdon, Plasma Physics via Computer Simulation (IOP Publishing, Bristol, U.K., 272 1991) p. 308.

273 


\section{List of Figure Captions}

276 Fig. 1. Flow chart of the three-dimensional particle model. First, initial conditions are set and

277 Maxwell's equations are solved by FDTD for the electromagnetic fields of $4.2 \mathrm{GHz}$ microwaves 278 to obtain a steady-state solution without plasma. Second, electrostatic PIC-MCC calculations are performed using the electrostatic field $\boldsymbol{E}_{\mathrm{ES}}$, the time-varying electric field of microwaves $\boldsymbol{E}_{\mathrm{EM}}$, and the magnetostatic fields of permanent magnets $\boldsymbol{B}_{\mathrm{st}}$, which is determined using ANSYS Emag $^{\mathrm{TM}}$ software. In the simulation, the power absorbed in the plasma $P_{0}$ is used as an input parameter.

Fig. 2. (Color online) Computational area and dimensions for calculations of the micro-ECR neutralizer, including the vacuum region for electron extraction: (a) $x-y$ plane $(z=1.0 \mathrm{~mm})$ at the antenna, (b) $z-x / y$ plane $(y / x=0.0 \mathrm{~mm})$, and (c) $x-y$ plane $(z=4.0 \mathrm{~mm})$ at the orifice plate. (d) Contour plots at the $z-y$ plane $(x=0.0 \mathrm{~mm})$ of the strength of the magnetic field of the ringshaped permanent magnets and the magnetic field lines in black, together with the thick lines in red representing the resonant magnetic field of $0.15 \mathrm{~T}$ for $4.2 \mathrm{GHz}$ microwaves.

291 Fig. 3. (Color online) Cross-sectional views of the time-averaged electron current density and 292 a few examples of its streamlines under the base case condition, where the xenon gas pressure 293 is $1 \mathrm{mTorr}$, the microwave frequency is $4.2 \mathrm{GHz}$, and the absorbed power is $0.3 \mathrm{~W}$. 
294 Fig. 4. (Color online) Two-dimensional distribution of the time-averaged $\boldsymbol{E} \times \boldsymbol{B}$ drift velocity in 295 the $z$ direction at the orifice plate $(z=4.0 \mathrm{~mm})$, together with circles in black representing the 296 boundaries of the ring-shaped permanent magnets and the four orifices. Note that the contours 297 in red represent the current density in the positive $z$ direction (from the plasma source to the 298 vacuum).

Fig. 5. (Color online) Scatter diagram of the positions for outgoing (red dots) and incoming (blue dots) electrons at the orifice plate $(z=4.0 \mathrm{~mm})$, together with circles in black representing the boundaries of the ring-shaped permanent magnets and the four orifices. Here, 1000 electrons are sampled at random upstream of the upper left orifice, and their trajectories are traced for a time span of 2000 microwave cycles.

Fig. 6. (Color online) Typical trajectory of a single electron extracted through one of the orifices together with vectors of the $\boldsymbol{E} \times \boldsymbol{B}$ drift velocity at some points, where the plane is set in such a way that the plane always passes through both the positions of the electron and the $z$-axis during

309 the electron tracking.

311 Fig. 7. (Color online) (a) Number of outgoing and incoming electrons passing through different 312 regions inside the orifices at $z=4.0 \mathrm{~mm}$ and (b) the definition of the regions. 


$$
\begin{gathered}
\text { Initial conditions } \\
n_{\mathrm{e} 0}, n_{\mathrm{i} 0}, T_{\mathrm{e} 0}, T_{\mathrm{i} 0}, P_{0}, \omega, p_{0}
\end{gathered}
$$

\section{FDTD}

Maxwell's Eqs. w/o plasma

$$
\begin{array}{r}
\nabla \times \boldsymbol{E}=-\frac{\partial \boldsymbol{B}}{\partial t} \\
\nabla \times \boldsymbol{B}=\mu_{0}\left(\boldsymbol{j}_{\mathrm{p}}+\varepsilon_{\mathrm{r}} \varepsilon_{0} \frac{\partial \boldsymbol{E}}{\partial t}\right) \\
\text { with } \boldsymbol{j}_{\mathrm{p}}=\mathbf{0}
\end{array}
$$

Magnetostatic field $B_{\text {st }}(\gg>B)$

FEM
PIC-MCC

Weighting of charge density

$$
\rho(\boldsymbol{x})=\Sigma q n S(\boldsymbol{x})
$$

$\downarrow$

Poisson's Eq. for $E_{\mathrm{ES}}$

$$
\Delta \phi=-\frac{\rho}{\varepsilon_{0}}, \quad \boldsymbol{E}_{\mathrm{ES}}=-\nabla \phi
$$

$\downarrow \boldsymbol{E}_{\mathrm{ES}}$

Moving particles \& BC $\Delta t_{\mathrm{i}}=40 \Delta t_{\mathrm{e}}$ $\boldsymbol{E}_{\mathrm{EM}}=\frac{\boldsymbol{E}_{0}}{\uparrow}{ }_{P_{\mathrm{abs}}=P_{0} ?}^{\cos (\omega t)} m \frac{d \boldsymbol{v}}{d t}=q\left(\boldsymbol{E}_{\mathrm{ES}}+\boldsymbol{E}_{\mathrm{EM}}+\boldsymbol{v} \times \boldsymbol{B}_{\mathrm{st}}\right)$
$\frac{d \boldsymbol{x}}{d t}=\boldsymbol{v}$

Monte Carlo collisions

$$
\boldsymbol{v} \stackrel{P}{\rightarrow} \boldsymbol{v}^{\prime}
$$


(a) $x$-y plane $(z=1 \mathrm{~mm})$

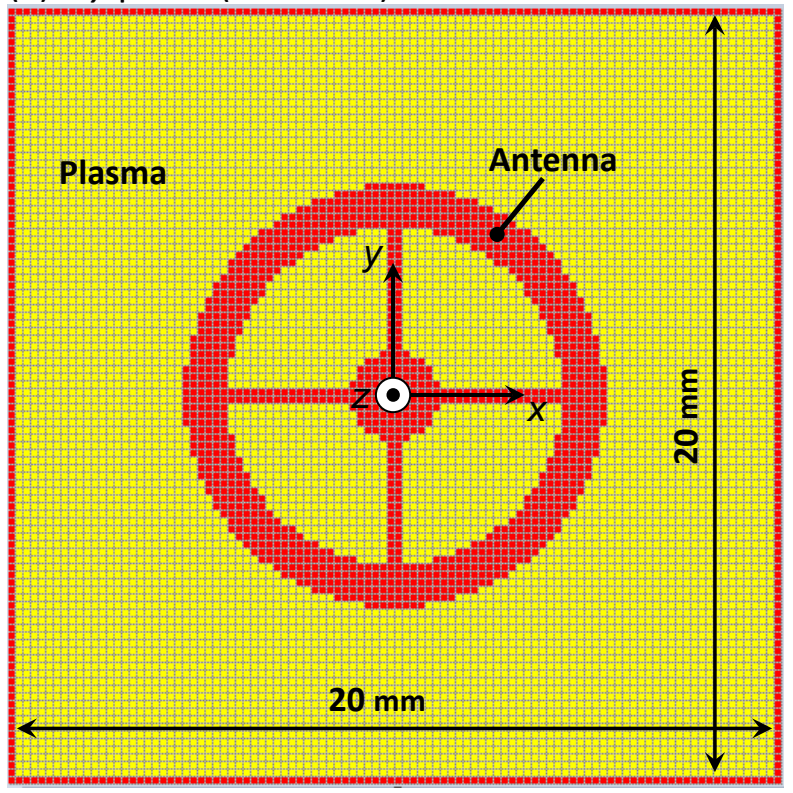

(c) $x$-y plane $(z=4 \mathrm{~mm})$

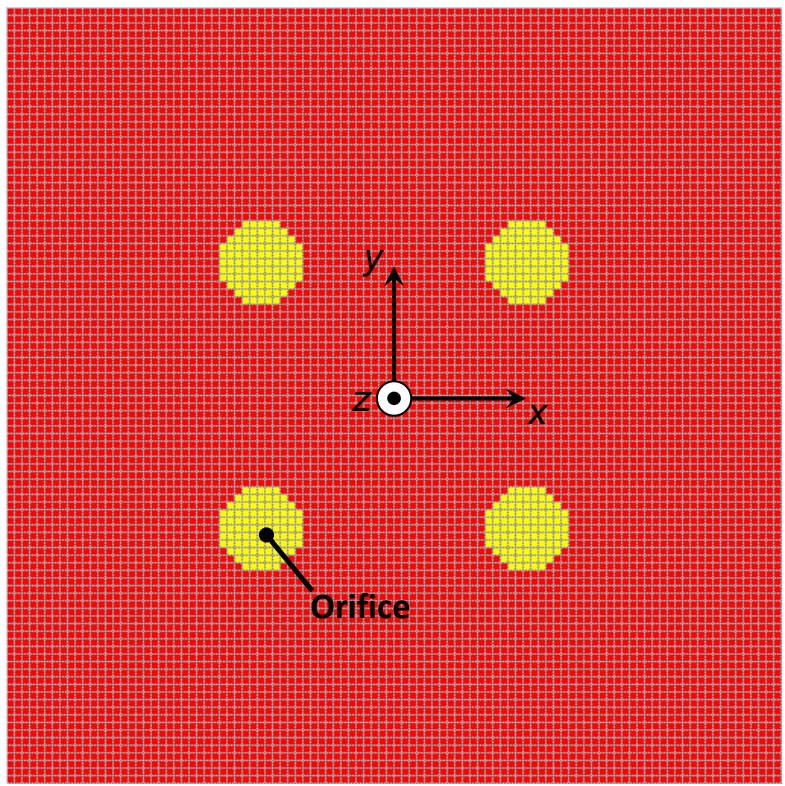
Fig. 2 (b) $z-x / y$ plane $(y / x=0 \mathrm{~mm})$

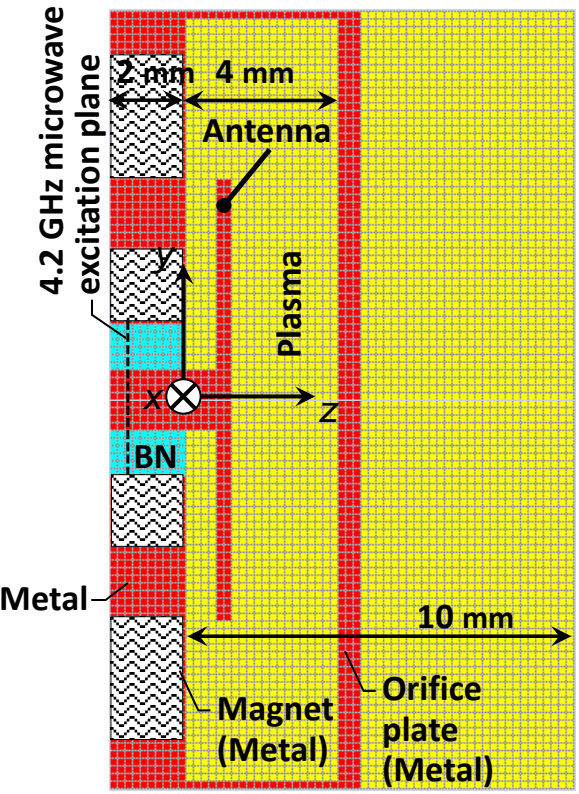

(d) Magnetic fields

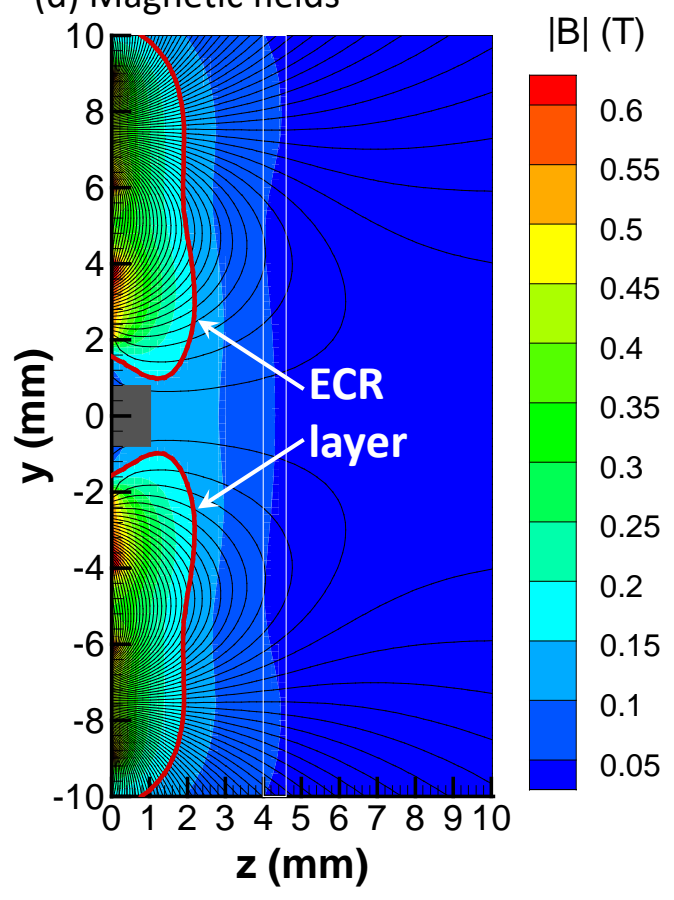




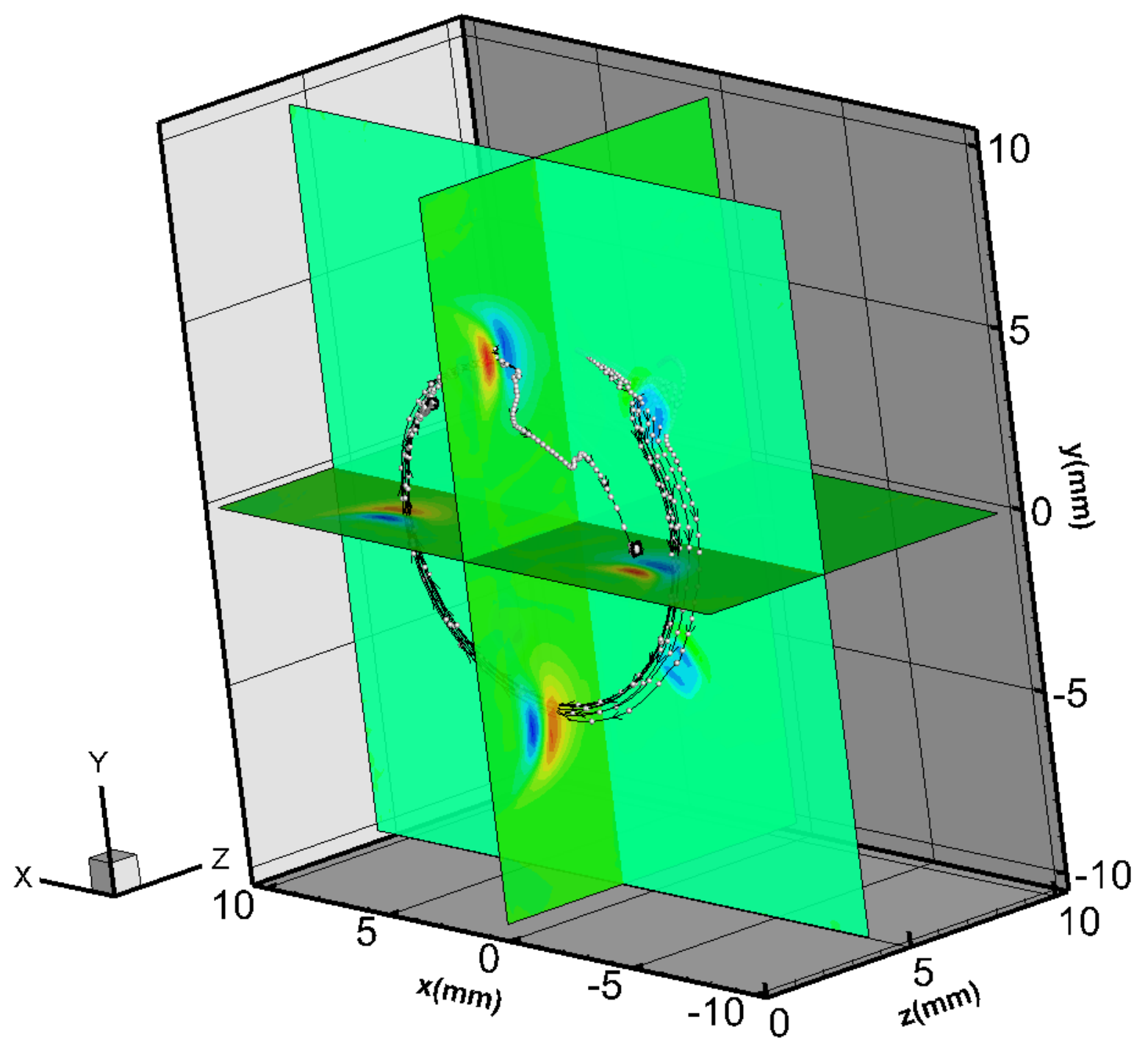

322

323

324

Fig. 3

325 


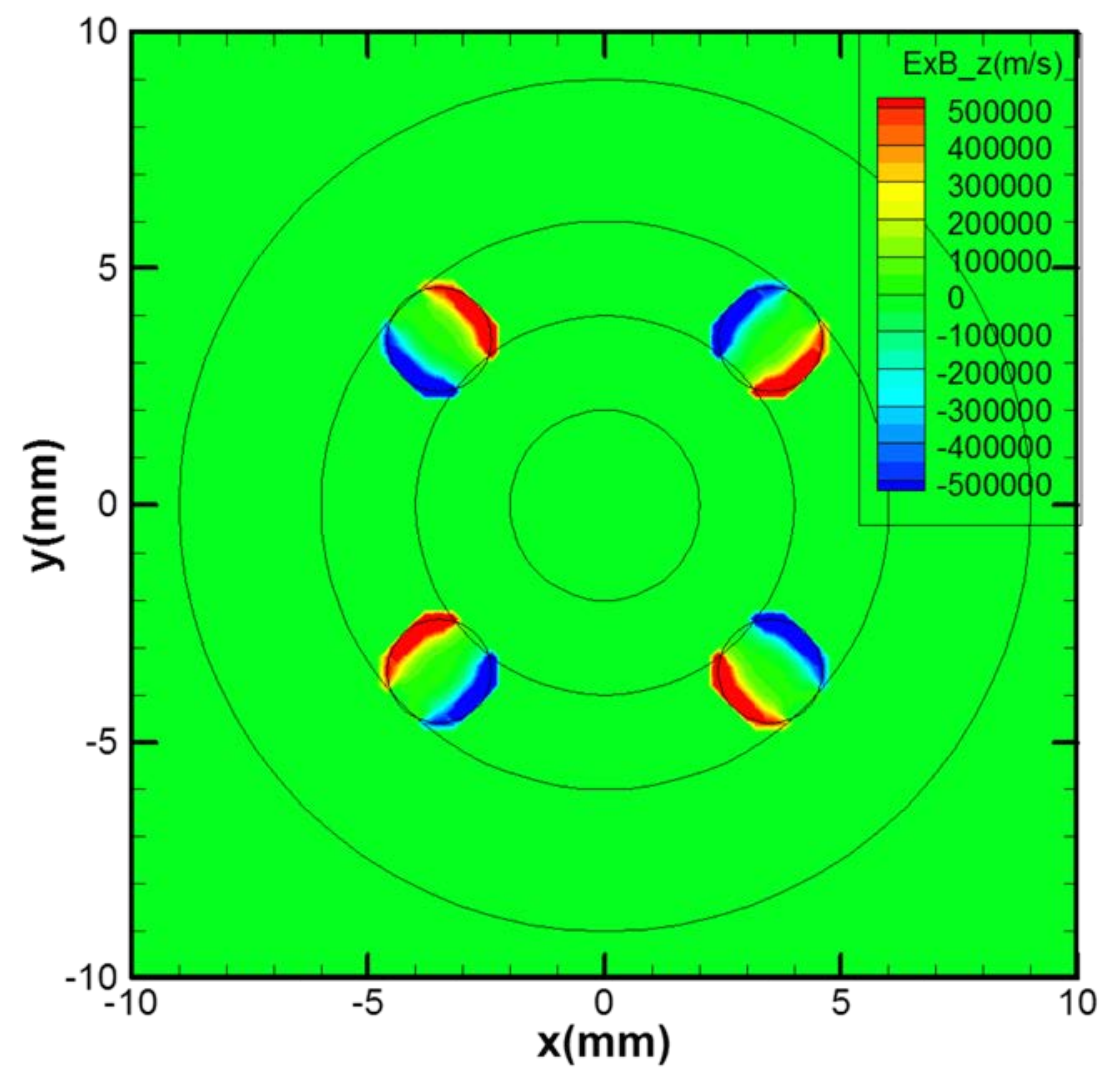

326

327

328

Fig. 4

329 


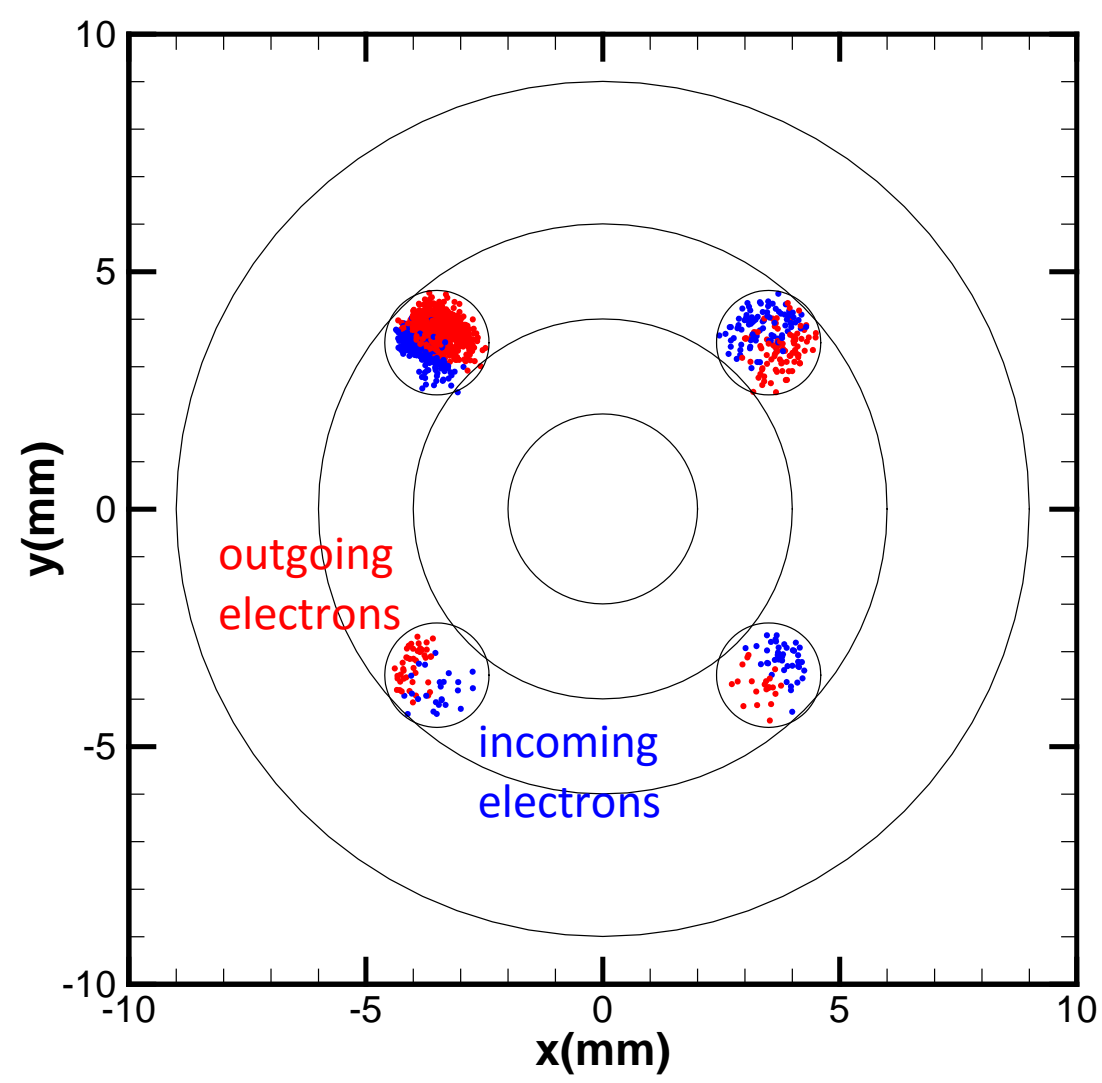

330

331

332

Fig. 5

333 


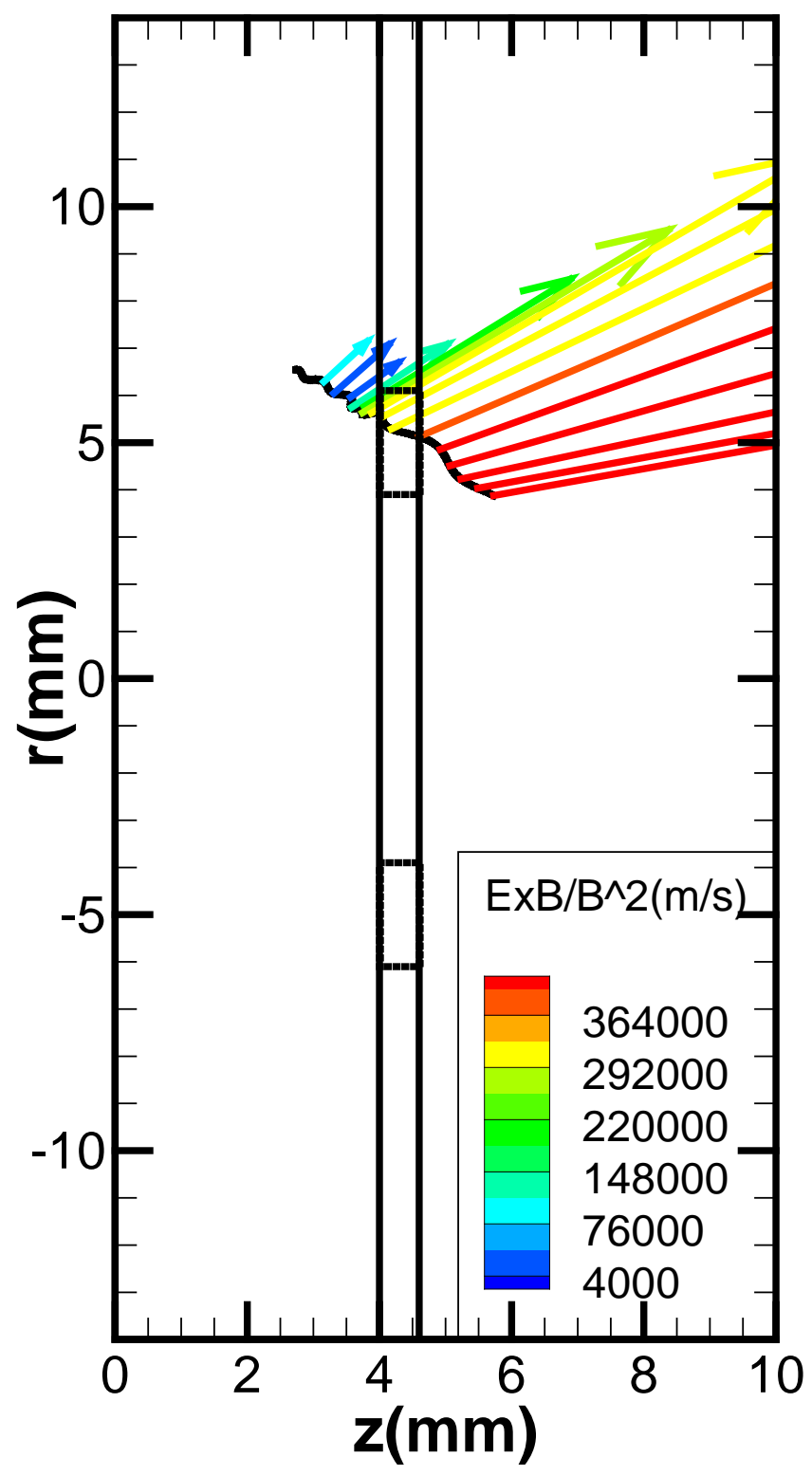

334

Fig. 6 
(a)

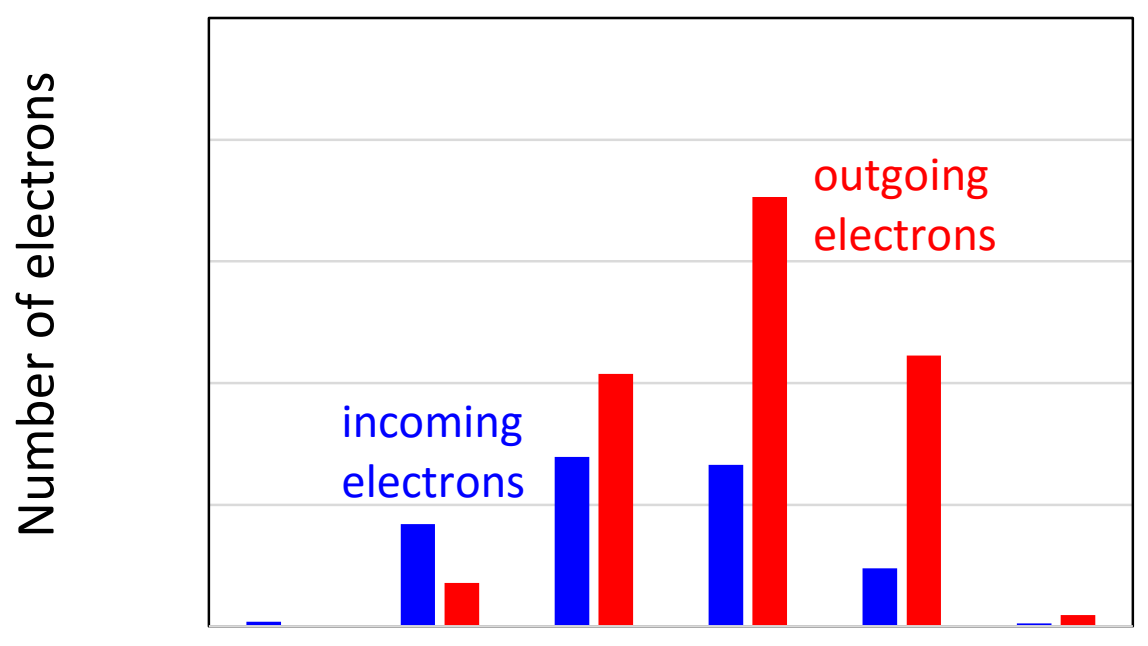

Angular degree

(b)

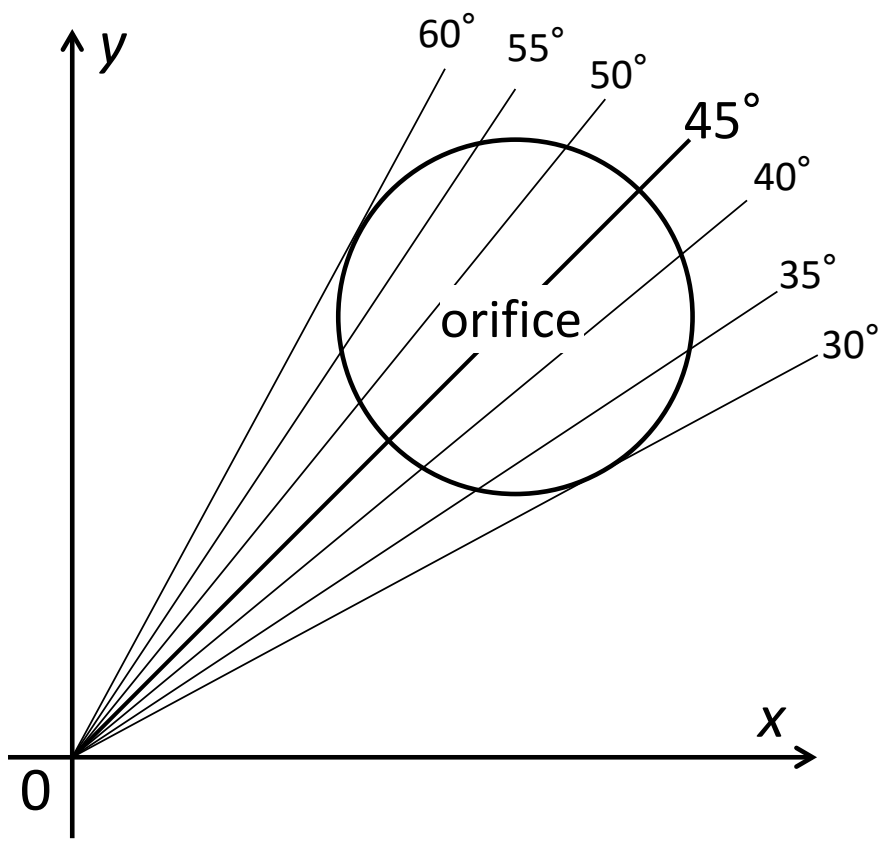

Fig. 7 